\title{
BMJ Open Comparison of strategies for monitoring and treating patients at the early phase of severe traumatic brain injury: the multicentre randomised controlled OXY-TC trial study protocol
}

Jean-Francois Payen (1) , ${ }^{1}$ Marion Richard, ${ }^{1}$ Gilles Francony, ${ }^{1}$ Gérard Audibert, ${ }^{2}$ Emmanuel L Barbier, ${ }^{1}$ Nicolas Bruder, ${ }^{3}$ Claire Dahyot-Fizelier, ${ }^{4}$ Thomas Geeraerts, ${ }^{5}$ Laurent Gergele, ${ }^{6}$ Louis Puybasset, ${ }^{7}$ Bernard Vigue, ${ }^{8}$ Kristina Skaare, ${ }^{9}$ Jean Luc Bosson, ${ }^{10}$ Pierre Bouzat ${ }^{11}$

To cite: Payen J-F, Richard M, Francony $\mathrm{G}$, et al. Comparison of strategies for monitoring and treating patients at the early phase of severe traumatic brain injury: the multicentre randomised controlled OXY-TC trial study protocol. BMJ Open 2020;10:e040550. doi:10.1136/ bmjopen-2020-040550

- Prepublication history and additional material for this paper are available online. To view these files, please visit the journal online (http://dx.doi org/10.1136/bmjopen-2020040550).

Received 18 May 2020 Revised 11 July 2020 Accepted 16 July 2020

Check for updates

(c) Author(s) (or their employer(s)) 2020. Re-use permitted under CC BY-NC. No commercial re-use. See rights and permissions. Published by BMJ.

For numbered affiliations see end of article.

Correspondence to

Professor Jean-Francois Payen; jfpayen@univ-grenoble-alpes.fr

\section{ABSTRACT}

Introduction Intracranial hypertension is considered as an independent risk factor of mortality and neurological disabilities after severe traumatic brain injury (TBI). However, clinical studies have demonstrated that episodes of brain ischaemia/hypoxia are common despite normalisation of intracranial pressure (ICP). This study assesses the impact on neurological outcome of guiding therapeutic strategies based on the monitoring of both brain tissue oxygenation pressure $\left(\mathrm{PbtO}_{2}\right)$ and ICP during the first 5 days following severe TBI.

Methods and analysis Multicentre, open-labelled, randomised controlled superiority trial with two parallel groups in 300 patients with severe TBI. Intracerebral monitoring must be in place within the first 16 hours posttrauma. Patients are randomly assigned to the ICP group or to the ICP $+\mathrm{PbtO}_{2}$ group. The ICP group is managed according to the international guidelines to maintain $I C P \leq 20 \mathrm{~mm} \mathrm{Hg}$. The ICP $+\mathrm{PbtO}_{2}$ group is managed to maintain $\mathrm{PbtO}_{2} \geq 20 \mathrm{~mm} \mathrm{Hg}$ in addition to the conventional optimisation of ICP. The primary outcome measure is the neurological status at 6 months as assessed using the extended Glasgow Outcome Scale. Secondary outcome measures include quality-of-life assessment, mortality rate, therapeutic intensity and incidence of critical events during the first 5 days. Analysis will be performed according to the intention-to-treat principle and full statistical analysis plan developed prior to database freeze. Ethics and dissemination This study has been approved by the Institutional Review Board of Sud-Est V (14-CHUG48) and from the National Agency for Medicines and Health Products Safety (Agence Nationale de Sécurité du Médicament et des produits de santé) (141435B31). Results will be presented at scientific meetings and published in peer-reviewed publications. The study was registered with ClinTrials NCT02754063 on 28 April 2016 (pre-results).

\section{Strengths and limitations of this study}

- This study will be a multicentre, randomised clinical trial to compare two therapeutic strategies based on early brain monitoring in patients with severe traumatic brain injury (TBI): intracranial pressure (ICP) alone versus ICP plus brain tissue oxygenation pressure $\left(\mathrm{PbtO}_{2}\right)$.

- This study is an open-label, non-blinded trial due to the nature of the intervention ( $\mathrm{PbtO}_{2}$ monitoring). However, outcome assessors and statisticians will be blinded to patient allocation.

- This study will collect extensive data during the first 5 days on admission. The maximum predefined delay of 16 hours post-TBI to allow inclusion represents a compromise that allows transportation to the participating site, patient screening and randomisation.

\section{INTRODUCTION}

Despite substantial efforts made over the past decades, the mortality rate following severe traumatic brain injury (TBI), as defined by an initial Glasgow Coma Scale (GCS) score of less than 9, remains within the range of $30 \%$ and $50 \%$, and only $20 \%$ of such patients will avoid lasting disabilities. ${ }^{1}$ TBI initiates a cascade of events that can lead to secondary brain damage or exacerbate the primary injury, and which develops hours to days after the initial insult. The concern over secondary brain damage is the focus of modern TBI management. The thresholds for irreversible tissue damage following TBI indicate a particular vulnerability of injured brain. ${ }^{2}$

The early recognition of secondary brain damage relies on neuromonitoring in critically ill sedated patients. International guidelines emphasise the use of intracranial 
pressure (ICP) monitoring following severe TBI, and the continuous calculation of cerebral perfusion pressure $(\mathrm{CPP}$, with $\mathrm{CPP}=$ mean arterial pressure $-\mathrm{ICP})$. To prevent brain ischaemia due to elevated ICP, maintenance of CPP between 60 and $70 \mathrm{~mm} \mathrm{Hg}$ and ICP below $20 \mathrm{~mm} \mathrm{Hg}$ is recommended. ${ }^{3}{ }^{4}$ Indeed intracranial hypertension, as defined by ICP values over $20 \mathrm{~mm} \mathrm{Hg}$, is considered as an independent risk factor of mortality and neurological disabilities. However, clinical studies have demonstrated that episodes of brain ischaemia/hypoxia are common despite optimisation of CPP or normalisation of ICP, and are independently associated with poorer patient outcome. ${ }^{5}$ Brain tissue hypoxia resulting from the imbalance between oxygen supply to the brain tissue and its utilisation is considered a major cause of the development of secondary brain damage, and thereby poor neurological outcome. ${ }^{6}$

Monitoring brain tissue oxygenation after TBI may help clinicians to initiate adequate reparative actions when episodes of brain ischaemia/hypoxia are identified. Measuring cerebral tissue oxygen tension can be safely and reliably achieved at the bedside using brain tissue oxygen pressure $\left(\mathrm{PbtO}_{2}\right)$ probes surgically inserted into the brain parenchyma. ${ }^{7} \mathrm{PbtO}_{2}$ measurements reflect the diffusion of dissolved plasma oxygen across the bloodbrain barrier. ${ }^{8} \mathrm{PbtO}_{2}$ values lower than $15 \mathrm{~mm} \mathrm{Hg}$ for more than $30 \mathrm{~min}$ were shown to be an independent predictor of unfavourable outcome and death. ${ }^{9} 10$ The aggressive treatment of low $\mathrm{PbtO}_{2}$ values has been associated with better outcome compared with standard ICP/ CPP-directed therapy in cohort studies of severely headinjured patients. ${ }^{11-13}$ However, others were unable to find similar benefits on patient outcome. ${ }^{14-17}$ All these studies were, however, uncontrolled, single-centre, and mostly retrospective. A randomised controlled trial recently showed that the information given by $\mathrm{PbtO}_{2}$ could help reduce the negative impact of brain tissue hypoxia with a trend towards more favourable outcome of patients in the $\mathrm{PbtO}_{2}$ treatment-guided group. ${ }^{18}$

The present programme will assess the impact of an early ICP and $\mathrm{PbtO}_{2}$ monitoring-based therapeutic strategy on neurological outcome in a randomised controlled trial. Each patient included in this study will enter into a 5-day intensive treatment modality to maintain ICP alone or ICP and $\mathrm{PbtO}_{2}$ within predefined values. The recent expert conference on algorithms for the management of patients with TBI with $\mathrm{PbtO}_{2}$ and ICP monitoring ${ }^{19}$ prompted us to publish the design of our ongoing randomised controlled trial OxyTC. An ancillary study will investigate the volume of brain lesions defined by abnormal values of mean diffusivity using magnetic resonance diffusion tension imaging (DTI) in the two groups of patients.

The primary objective of the study is to determine whether early optimisation of brain oxygenation during the first 5 days after severe non-penetrating TBI improves neurological outcome at 6 months. Secondary objectives are to determine whether early optimisation of brain oxygenation improves survival at day 28, quality of life at 6 and 12 months, and neurological outcome at 12 months after TBI, and affects therapeutic intensity and incidence of critical events during the first 5 days of the intensive care unit (ICU) stay. The ancillary objective is to determine whether the volume of brain lesions after injury as measured with multiparametric, quantitative MRI on average is reduced with a therapeutic strategy based on $\mathrm{PbtO}_{2}$ and ICP measurements.

\section{METHODS AND ANALYSIS}

\section{Trial design}

The OxyTC trial is a national, multicentre, open-labelled, randomised controlled superiority trial with two parallel groups and 1:1 allocation ratio. Figure 1 shows the study design and flow of the OxyTC trial.

\section{Study setting}

The OxyTC trial includes 22 tertiary referral centres within university hospitals (Grenoble, Saint-Etienne, Rennes, Clermont-Ferrand, La Reunion, Bordeaux, Nancy, Marseille, Besançon, Lille, Nice, Paris PitiéSalpêtrière, Poitiers, Rouen, Strasbourg, Dijon, Caen, Toulouse, Nimes, Angers) and non-university hospitals (Annecy, Toulon). Each centre was chosen on documentation for patient availability and experience in care management of patients with severe TBI .

\section{Study population}

Patients are included if they meet the following criteria: aged between 18 and 75 years, admitted for a severe non-penetrating TBI with GCS score 3-8 and motor component $1-5$, require ICP monitoring and continuous sedation/analgesia for more than 48 hours, and are mechanically ventilated with stable condition. Intracerebral monitoring (ICP with or without $\mathrm{PbtO}_{2}$ ) must be in place within the first 16 hours from injury. Patients may have extracranial lesions but not quadriplegia. Frenchspeaking or English-speaking patients must be affiliated to either the French social security system or other social security system of another european member state, and give their written informed consent through legal surrogates or relatives.

Patients are excluded if they have one of the following criteria: penetrating head injury, GCS score 3 with bilateral fixed dilated pupils, decompressive craniectomy and no repositioning of the bone flap after subdural hematoma evacuation surgery prior to enrolment, contraindication to ICP and/or $\mathrm{PbtO}_{2}$ monitoring, persistent haemodynamic or respiratory instability despite treatments, body temperature $<34^{\circ} \mathrm{C}$ at randomisation, life expectancy $<24$ hours, cardiac arrest at the initial presentation, associated quadriplegia, neuropsychiatric comorbidities that could interfere with the assessment of outcomes at 6 and 12 months, consent refusal, participation in another therapeutic study with written consent, impossibility to follow-up, ischaemic stroke after carotid 


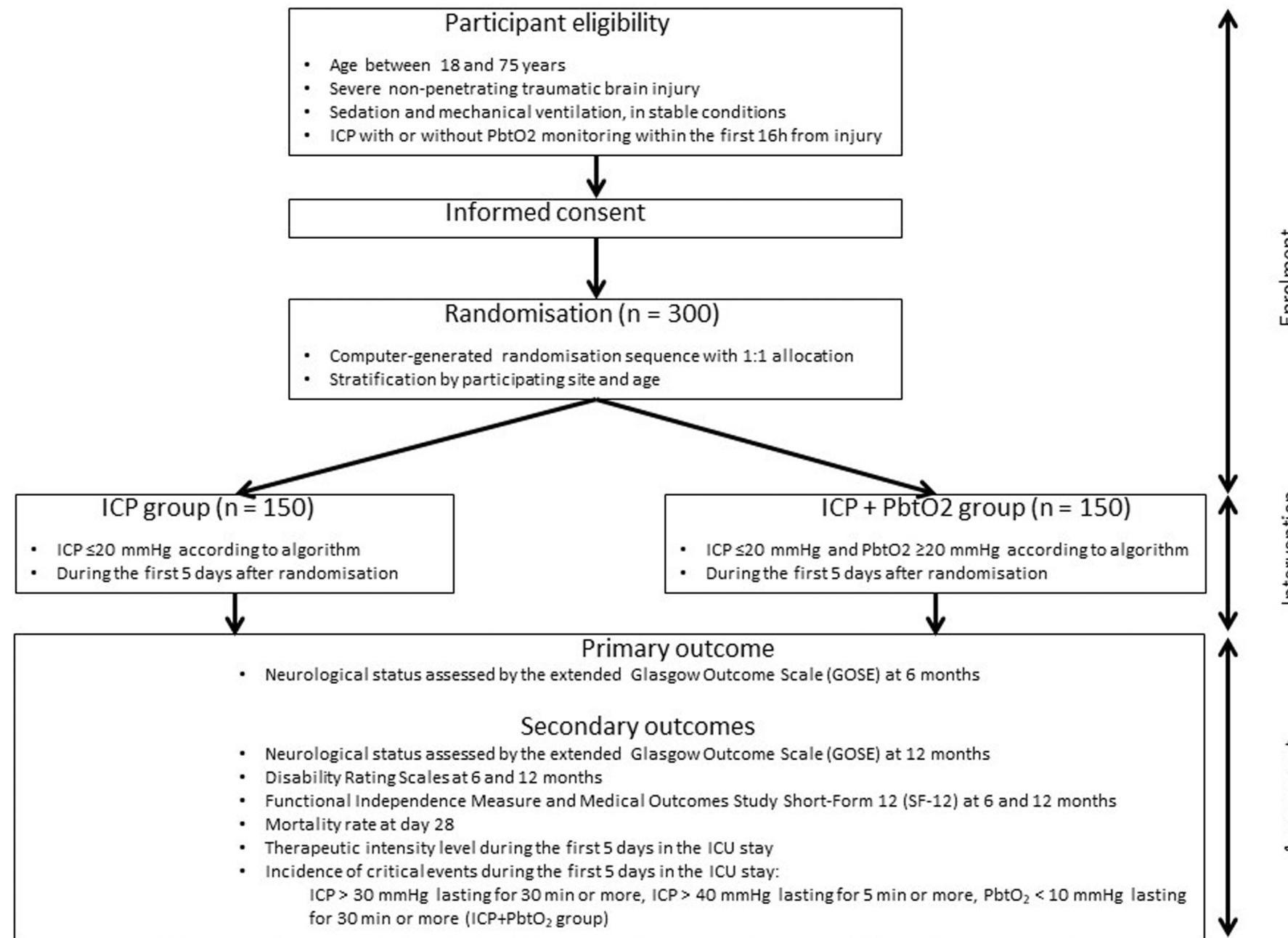

Figure 1 Study design and flow of the Oxy-TC trial. ICP, intracranial pressure; ICU, intensive care unit; $\mathrm{PbtO}_{2}$, brain tissue oxygen pressure.

arterial dissection, incapacitated patients in accordance with article L 1121-5 to L1121-8 of the French public health code: pregnant or breastfeeding women, persons deprived of liberty for civil reasons and those deprived of liberty on criminal charges, persons under psychiatric treatments and persons residing in a public health or social institution, adults under guardianship or adults permanently unable to express their wishes.

Enrolled patients are withdrawn for any of the following reasons: inability to measure ICP and/or $\mathrm{PbtO}_{2}$ during at least 48 hours, due to failure to insert catheter(s), permanent contraindication to intracerebral monitoring, defective or unavailable material, withdrawal of consent or consent to continue not granted, any serious adverse event or protocol deviation obliging, according to the in-charge physician, their exclusion, ischaemic stroke after traumatic carotid or vertebrobasilar artery dissection on CT scan at day 2.

\section{Randomisation and blinding}

Patients with informed consent for participation who fulfil the inclusion criteria are randomised. Randomisation is performed by the site investigator or by the research coordinator through a dedicated password protected, SSL-encrypted website (Medsharing, Fontenay-sous-Bois, France) to allow concealed computer-generated random allocation. Protocol allocation is stratified by participating site and age ( $<50$ years and $\geq 50$ years), and patients are assigned to ICP management (ICP group) or ICP and $\mathrm{PbtO}_{2}$ management (ICP $+\mathrm{PbtO}_{2}$ group) in a 1:1 ratio.

This trial is an open-label, non-blinded trial for the patient and the in-charge physician due to the nature of the intervention (presence or not of $\mathrm{PbtO}_{2}$ monitoring). Blinded assessment of the primary outcome will be performed at the coordinating centre and ensured by the entering of no identifying data or allocated group into the database. Statistical analyses will be based on blinded data.

\section{Intervention}

Patients eligible for inclusion are randomly assigned to the ICP group or to the ICP $+\mathrm{PbtO}_{2}$ group. The two groups of patients are similarly managed according to first-line treatments aimed at preventing any source of secondary brain damage (level 1): continuous sedation and analgesia, mechanical ventilation in normocapnia 
ICP group

$\mathrm{ICP} \leq 20 \mathrm{mmHg}$

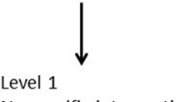

No specific interventions

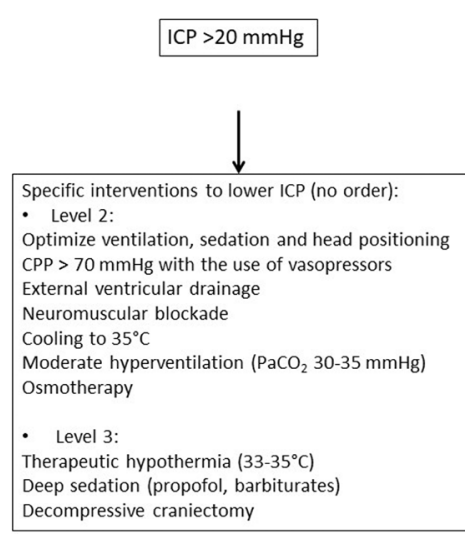

Figure 2 Algorithms for the therapeutic strategies for the ICP group. CPP, cerebral perfusion pressure; ICP, intracranial pressure.

and normoxia, CPP 60-70 mm $\mathrm{Hg}$, euvolaemia, normal levels of serum glucose and sodium, no anaemia and body temperature $36^{\circ} \mathrm{C}-38^{\circ} \mathrm{C}$. ${ }^{4}$ Patients are placed in the supine position with $15^{\circ}$ head-up tilt.

The ICP group is treated according to international guidelines. If ICP $>20 \mathrm{~mm} \mathrm{Hg}$, treatments are gradually introduced (level 2 and level 3 if needed): deep level of sedation/analgesia, vasopressors, moderate hyperventilation, osmotic agents, external ventricular drainage, muscle relaxants, therapeutic hypothermia, decompressive craniectomy and barbiturates. The ICP $+\mathrm{PbtO}_{2}$ group is treated as above for the ICP group but also to maintain
$\mathrm{PbtO}_{2}$ values over $20 \mathrm{~mm} \mathrm{Hg} .{ }^{10} 20$ The optimisation of $\mathrm{PbtO}_{2}$ is thus in addition to that of ICP, and includes procedures directed at increasing blood oxygen supply to the brain: CPP increase, cardiac output optimisation, $\mathrm{PaO}_{2}$ increase, normocapnia and blood transfusion. The treatment algorithms according to allocated therapeutic strategy are shown in figures 2 and 3.

The maximal therapeutic intensity after severe TBI is usually observed during the first week of the ICU stay. ${ }^{21}$ Given an expected stay of 10-20 days in the ICU after severe TBI and the necessity to reach the highest adherence to the study protocol, data are collected during the first 5 days following TBI and the initiation of one of two therapeutic algorithms. Beyond this, patients are treated according to guidelines ${ }^{34}$ in each participating centre, and the use of $\mathrm{PbtO}_{2}$ and ICP monitoring is left at the discretion of the in-charge physician.

\section{Study outcomes}

The primary outcome measure is the neurological status at 6 months after TBI as assessed using the extended Glasgow Outcome Scale (GOSE).$^{22}$ Treatment success is defined as the proportion of patients with unfavourable outcome, that is, GOSE score of 1 (death) to 4 (upper severe disability), is reduced by $30 \%$ in the $\mathrm{ICP}+\mathrm{PbtO}_{2}$ group compared with the ICP group. Questionnaire scoring is conducted during a telephone interview by trained central outcome assessors who are blinded to the treatment arm.

Secondary outcomes are neurological outcome according to the GOSE at 12 months post-trauma and

\section{$\mathrm{ICP}+\mathrm{PbtO}_{2}$ group}

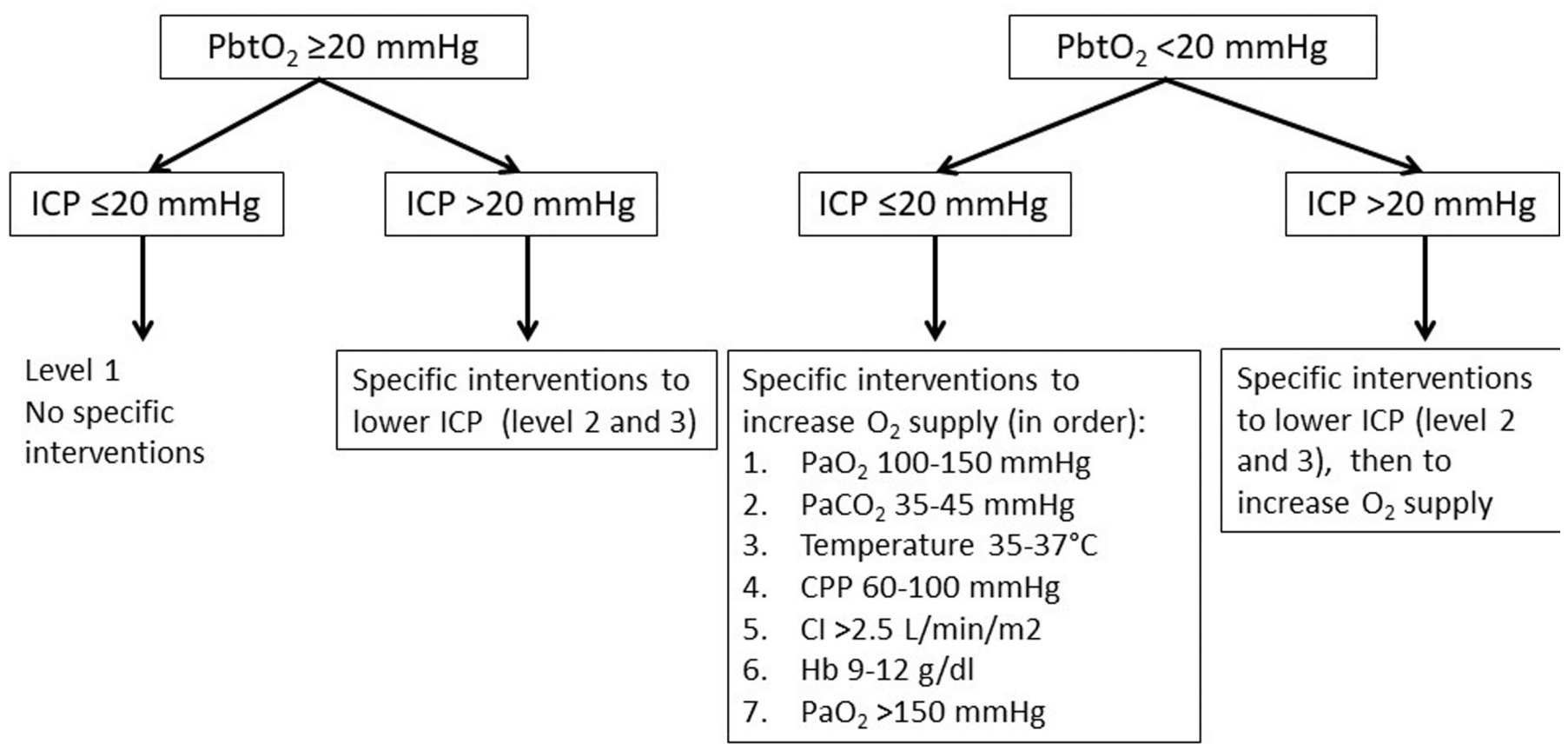

Figure 3 Algorithms for the therapeutic strategies for the ICP $+\mathrm{PbtO}_{2}$ group. $\mathrm{Cl}$, cardiac index; CPP, cerebral perfusion pressure; $\mathrm{Hb}$, haemoglobin; ICP, intracranial pressure; PbtO2, brain tissue oxygen pressure. 
the Disability Rating Scale (DRS) at 6 and 12 months post-trauma, quality-of-life assessment: Functional Independence Measure and Medical Outcomes Study ShortForm 12 at 6 and 12 months post-trauma, mortality rate at day 28, therapeutic intensity during the first 5 days of the ICU stay as reflected by the number of level 2 and level 3 treatments to treat elevated ICP, and incidence of critical events during the first 5 days of the ICU stay as defined by ICP $>30 \mathrm{~mm} \mathrm{Hg}$ lasting for $30 \mathrm{~min}$ or more, ICP $>40 \mathrm{~mm} \mathrm{Hg}$ lasting for $5 \mathrm{~min}$ or more, $\mathrm{PbtO}_{2}<10 \mathrm{~mm}$ $\mathrm{Hg}$ lasting for $30 \mathrm{~min}$ or more $\left(\mathrm{ICP}+\mathrm{PbtO}_{2}\right.$ group). For the ancillary study (MRI), the volume of cerebral lesions with abnormal mean diffusivity values is determined using diffusion tensor MR imaging between days 6 and 10 after severe TBI.

\section{Data collection, data monitoring and adverse events}

At each participating site, data are collected and entered into the web-based electronic case report form (eCRF) (Medsharing, Fontenay-sous-Bois, France) by trial or clinical monitors (clinical research associates) under the supervision of the site principal investigators. The trial database will be created from the eCRF. Trained research coordinators monitor data collection. The study collects demographic, baseline information at randomisation, intracerebral monitoring measured hourly on day 1 to day 5 , extracerebral information on vital signs and therapies measured every 6 hours on day 1 to day 5 , biological data measured every 12 hours on day 1 to day 5 , adverse events during the ICU stay and functional outcome at 6 and 12 months.

Several procedures to ensure data quality and protocol standardisation are in place to help minimise bias. They include: (1) a full-day investigator meeting for all principal investigators and lead research coordinators prior to study commencement to ensure consistency in procedures; (2) a site initiation visit conducted prior to site activation to provide protocol training for all site staff; (3) if needed, training sessions in $\mathrm{PbtO}_{2}$ management prior to site activation; (4) a detailed Oxy-TC operations manual provided for the investigators and research coordinators; (5) one or more visit as required from the project manager to each investigating site during the recruitment period to verify data sources and provide retraining as necessary; (6) centralised maintenance of the electronic database at Grenoble Clinical Research Centre and (7) for the MRI substudy, a validation of all participating sites including a quality check of MRI data, patient transport and MRI transfer procedures prior to site activation.

An independent data and safety monitoring committee (DSMC), comprising five experts in clinical trials, pharmacology, neurosurgery and intensive care medicine, was established before the first patients were enrolled. The DSMC advise the trial management committee as to whether the study should continue based on findings from the monitoring process and serious adverse events reported. The DSMC meet on the enrolment of every 50 randomised patients and on sponsor demand in case of suspected unexpected serious adverse events to $\mathrm{PbtO}_{2}$ probes or unexpected frequency of expected events. Meetings take place by videoconference or callconference.

It is recognised that the patient population with severe TBI will experience a number of common aberrations in laboratory values, as well as clinical signs and symptoms due to the severity of the underlying disease and the impact of standard therapies. These will not necessarily constitute an adverse event unless they require serious intervention or are considered a concern to the investigators based on their clinical judgement. Adverse events expected during this clinical trial are:

- Related to severe TBI: death, severe neurological disability, organ failure during the ICU stay, hydrocephalus with dysfunction of external ventricular drainage and ICU-acquired complications, that is, ventilatorassociated pneumonia, colonisation of central venous catheters, urinary tract infection, positive blood cultures, venous thromboembolism, gastroduodenal haemorrhage, neuromyopathy.

- Related to insertion of intracerebral catheters: intracerebral haematoma, meningitis and cerebral thrombophlebitis, local infection, probe dysfunction.

Any adverse events classed as serious, that is, causing death, or requiring subsequent extension of ICU stay or intervention(s) not planned in the therapeutic algorithm, are reported to the sponsor (person responsible for the safety of the clinical trial).

\section{Statistical considerations}

According to the available literature, ${ }^{123} 24$ including the most recent data, ${ }^{25}$ the rate of unfavourable neurological outcome (death and severe disability) following severe TBI is $55 \%$ (ICP group). Assuming a two-sided alpha risk of 0.05 , the enrolment of two equally sized groups (148 patients per group) will have $80 \%$ power to detect a $30 \%$ reduction in relative risk of unfavourable neurological outcome at 6 months, that is, an absolute reduction of $17 \%$ in the rate of unfavourable neurological outcome. A total of 300 patients have to be recruited for the study. No interim analysis is planned.

The full statistical analysis plan will be developed prior to database freeze. Data will be expressed as mean and $95 \%$ CIs, or median and IQRs where appropriate. Comparisons will be conducted on an intention-to-treat basis. Missing data will be described and compared in terms of incidence between the two groups of patients. If the primary outcome is missing, data will be replaced as follows: no replacement if data are missing for less than $5 \%$ of patients, or using multiple imputation should data be missing from between $5 \%$ and $15 \%$ of patients. Patients not receiving the allocated protocol during the 5-day study, for example, early death or discharge from the ICU within the first 5 days, are followed up for their outcome and analysed on an intention-to-treat principle.

Baseline variables will be compared using $\mathrm{X}^{2}$ tests for equality of proportions, Student's t-test for normally distributed outcomes and Wilcoxon rank-sum tests 
otherwise. The primary outcome measure will be analysed using $\mathrm{X}^{2}$ tests. Data from the GOSE and DRS will be analysed using two-way analysis of variance for repeated measurements (M6/M12). Survival at day 28 will be tested with the Kaplan-Meier model and Cox model adjusted for age and centre. The results will be presented according to the Consolidated Standards of Reporting Trials guidelines for parallel group randomised trials. ${ }^{26}$ Statistical significance will be declared when $\mathrm{p} \leq 0.05$.

\section{Study timescale}

2014-2016: protocol design and approval from the Institutional Review Board and from the National Agency for Medicines and Health Products Safety (ANSM, Agence Nationale de Sécurité du Médicament et des produits de santé), eCRF and randomisation system building, and quality control for the MRI ancillary study. Please see administrative information in online supplementary file.

2016-2021: inclusion of patients. The first patient was recruited on 15 June 2016.

2022: 1-year follow-up of patients.

2023: cleaning and closure of the database, data analyses, manuscript writing and publication.

\section{Patient and public involvement}

No patient involved

\section{ETHICS AND DISSEMINATION}

The protocol was approved by the Institutional Review Board of Sud-Est V on 3 December 2014 (14-CHUG-48) and by the National Agency for Medicines and Health Products Safety (ANSM) on 3 February 2015 (141 435B31). Patients with severe TBI do not have the capacity to provide informed consent. Consent is, therefore, obtained through the signing of a consent form by a patient's relative or legal surrogate (person legally allowed to give consent on behalf of the patient) following a verbal presentation of the study provided by the site investigator, the reading through of the consent form, and answering of any questions.

In the event where the legal surrogate or patient's relative cannot attend the hospital to sign the consent form on time, or cannot be immediately determined or contacted, the patient may be enrolled with the signature of the site investigator, in accordance with French legislation (Procedural Authorisation). Consent from the legal surrogate or patient's relative is obtained later and as soon as possible. At such later stages, any patient whose recovery permits their decision making and communication is asked directly for their consent. A specific signed consent covering the main clinical trial and the ancillary MRI study must be obtained in the same conditions.

Results of this study will be presented at national and international meetings and published in peerreviewed journals. Patients will not be individually notified regarding the results of the study. The principal publication from the study will be in the name of the
Oxy-TC investigators with full credit assigned to all active, collaborating investigators, research coordinators and institutions.

\section{DISCUSSION}

Compared with normal ICP, elevated ICP is known to be closely linked with higher mortality and disability rates. ${ }^{27}$ Longer duration and greater intensity of episodes of high ICP were also associated with worse outcome in adults and children. ${ }^{28}$ However, controlling ICP does not guarantee a good outcome, as shown with recent trials. ${ }^{249}{ }^{20}$ Instead, there is a growing interest in controlling brain oxygenation with the monitoring of brain hypoxia becoming part of the standard monitoring of TBI patients, that is, situations where oxygen supply to the brain and oxygen consumption are imbalanced such as arterial hypertension, low cardiac output, hypocapnia, systemic hypoxia, anaemia and hyperthermia. The diagnostic accuracy of near-infrared spectroscopy is limited in patients with $\mathrm{TBI}^{31}$ and jugular venous oxygen saturation monitoring has been gradually replaced with $\mathrm{PbtO}_{2}$ in clinical practice.

The usefulness of $\mathrm{PbtO}_{2}$ monitoring in patients with TBI was recently demonstrated in a randomised trial (Brain Oxygen Optimization in Severe Traumatic brain injury, BOOST-2) where information provided by $\mathrm{PbtO}_{2}$ appeared to help reduce the proportion of time spent with brain hypoxia. ${ }^{18}$ Although that study was not powered for clinical efficiency, a trend towards lower mortality and better outcome was observed in the ICP $+\mathrm{PbtO}_{2}$ group compared with the ICP group. These results prompted the authors to launch a phase III trial (BOOST-3; NCT03754114) that is open for recruitment since August 2019 using a similar methodological approach and primary outcome as the Oxy-TC trial. Both trials will provide evidence regarding the potential benefit or harm of invasive monitoring of brain oxygenation after severe TBI.

There are some limitations with the Oxy-TC protocol. First, no specific protocol directed at lowering ICP to $\leq 20 \mathrm{~mm} \mathrm{Hg}$ is recommended. The choice of treatments is left to the discretion of the in-charge physician providing the reporting of the chosen therapies and results on ICP. In the cited expert consensus conference, ${ }^{19}$ the ICP threshold at which treatment should be triggered was set at $22 \mathrm{~mm} \mathrm{Hg}$, a value not widely used in clinical practice. Second, patients with various brain lesions can be recruited provided they meet inclusion criteria. Some lesions might benefit from additional monitoring such as large contusions, while other lesions such as diffuse axonal injuries might not. However, the randomisation process should ensure the equal distribution of types of brain lesions across the two groups of patients. Third, patients aged over 75 years are excluded from this study. Indeed, the determination of TBI-related neurological outcome in the elderly can be affected by pre-existing cognitive alterations. We stratified the study population by age $(<50$ years and $\geq 50$ years) to prevent an uncontrolled 
effect of age. Fourth, the maximum predefined delay of 16 hours post-TBI to allow inclusion may be viewed as too long. While accounting for the necessity to initiate patient management at the early phase of TBI, this delay represents a compromise that allows transportation to the participating site, patient screening and randomisation. Fifth, quantitative MRI is an ancillary study. Initially, the quantification of brain lesion volume using DTI was the primary outcome of this study (see above). However, difficulties to access MRI during weekdays, some enrolled patients being unable to access MRI facilities between days 6 and 10 after TBI and/or technical problems relating to quality of MR imaging resulted in a large number of missing data. In agreement with the French legal authorities, the primary outcome was, therefore, changed on 7 February 2018 to compare the neurological outcome at 6 months between the two strategies. Sixth, we estimate that 300 patients will be needed to show a $30 \%$ relative reduction of unfavourable outcome in the ICP $+\mathrm{PbtO}_{2}$ group. This size effect is in line with other cohort studies. ${ }^{1-13} \mathrm{In}$ the BOOST-2 trial, a trend towards reduced mortality and better outcome in the ICP $+\mathrm{PbtO}_{2}$ group was observed with 53 patients in each group.

In conclusion, data obtained from this study may show that early monitoring of brain oxygenation using $\mathrm{PbtO}_{2}$ in addition to ICP monitoring can improve neurological outcome in patients with TBI. If this is the case, hundreds of patients per year could benefit from the combined monitoring. The resulting change of even just a few of these patients from an unfavourable to a favourable neurological outcome would also result in large savings for the healthcare system.

\section{Author affiliations}

${ }^{1}$ Department of Anaesthesia and Intensive Care, Univ. Grenoble Alpes, Centre Hospitalier Universitaire Grenoble Alpes, Grenoble Institut des Neurosciences, INSERM, U1216, Grenoble, France

${ }^{2}$ Department of Anaesthesia and Intensive Care, Lorraine University, Nancy University Hospital, Nancy, France

${ }^{3}$ Department of Anaesthesiology and Intensive Care, Aix-Marseille University, Assistance Publique - Hôpitaux de Marseille, Marseille, France

${ }^{4}$ Department of Anaesthesia and Intensive Care, Poitiers University Hospital and Poitiers Hospital, Pharmacology of antimicrobial agents, INSERM U1070, Poitiers, France

${ }^{5}$ Department of Anaesthesia and Intensive Care, Toulouse University Hospital and Toulouse 3-Paul Sabatier University, Toulouse, France

${ }^{6}$ Department of Intensive care, Ramsay Sante, Hopital Privé de la Loire, SaintEtienne, France

${ }^{7}$ Department of Anaesthesia and Critical Care, Sorbonne University, GRC 29, AP-HP, DMU DREAM, Pitié-Salpêtrière Hospital, Paris, France

${ }^{8}$ Department of Anaesthesia and Intensive care, Centre Hospitalier Universitaire de Bicêtre, Assistance Publique - Hopitaux de Paris, Le Kremlin Bicêtre, France ${ }^{9}$ Department of Public Health, Univ. Grenoble Alpes, CHU Grenoble Alpes, Grenoble, France

${ }^{10}$ TIMC IMAG, Centre Hospitalier Universitaire Grenoble Alpes, Grenoble, France

${ }^{11}$ Centre Hospitalier Universitaire de Grenoble, Grenoble, France

Contributors JFP conceived the study. GF, GA, EB, NJB, CDF, TG, LG, LP, BV and PB initiated the study design. JFP, MR, EB and PB helped with implementation. JFP and $E B$ are grant holders. KS and JLB provided statistical expertise in clinical trial design, and JLB is conducting the primary statistical analysis.
Funding This study is supported by a grant from the French National Program for Clinical Research (RCB 2014-A01674-43) and by an unrestricted grant from Integra Lifesciences (38RC14.039).

Disclaimer These funding sources have no role in the design of this study and will not have any role during its execution, analyses, interpretation of the data, or decision to submit results. Centre Hospitalier Universitaire Grenoble Alpes, Grenoble, France (Mrs M Sorrentino, Director General).

Competing interests No.

Patient consent for publication Not required.

Provenance and peer review Not commissioned; externally peer reviewed.

Open access This is an open access article distributed in accordance with the Creative Commons Attribution Non Commercial (CC BY-NC 4.0) license, which permits others to distribute, remix, adapt, build upon this work non-commercially, and license their derivative works on different terms, provided the original work is properly cited, appropriate credit is given, any changes made indicated, and the use is non-commercial. See: http://creativecommons.org/licenses/by-nc/4.0/.

ORCID iD

Jean-Francois Payen http://orcid.org/0000-0001-8400-3839

\section{REFERENCES}

1 Roozenbeek B, Maas AIR, Menon DK. Changing patterns in the epidemiology of traumatic brain injury. Nat Rev Neurol 2013;9:231-6.

2 Cunningham AS, Salvador R, Coles JP, et al. Physiological thresholds for irreversible tissue damage in contusional regions following traumatic brain injury. Brain 2005;128:1931-42.

3 Brain Trauma Foundation, American Association of Neurological Surgeons, Congress of Neurological Surgeons. Guidelines for the management of severe traumatic brain injury. IX. cerebral perfusion thresholds. J Neurotrauma 2007;24:S59-64.

4 Geeraerts T, Velly L, Abdennour L, et al. Management of severe traumatic brain injury (first 24hours). Anaesth Crit Care Pain Med 2018;37:171-86.

5 Oddo M, Levine JM, Mackenzie L, et al. Brain hypoxia is associated with short-term outcome after severe traumatic brain injury independently of intracranial hypertension and low cerebral perfusion pressure. Neurosurgery 2011;69:1037-45.

6 Bouzat P, Sala N, Payen J-F, et al. Beyond intracranial pressure: optimization of cerebral blood flow, oxygen, and substrate delivery after traumatic brain injury. Ann Intensive Care 2013;3:23.

7 Haitsma IK, Maas AIR. Advanced monitoring in the intensive care unit: brain tissue oxygen tension. Curr Opin Crit Care 2002;8:115-20.

8 Rosenthal G, Hemphill JC, Sorani M, et al. Brain tissue oxygen tension is more indicative of oxygen diffusion than oxygen delivery and metabolism in patients with traumatic brain injury. Crit Care Med 2008;36:1917-24.

9 van den Brink WA, van Santbrink H, Steyerberg EW, et al. Brain oxygen tension in severe head injury. Neurosurgery 2000;46:868-76.

10 Chang JJJ, Youn TS, Benson D, et al. Physiologic and functional outcome correlates of brain tissue hypoxia in traumatic brain injury. Crit Care Med 2009;37:283-90.

11 Stiefel MF, Spiotta A, Gracias VH, et al. Reduced mortality rate in patients with severe traumatic brain injury treated with brain tissue oxygen monitoring. J Neurosurg 2005;103:805-11.

12 Narotam PK, Morrison JF, Nathoo N. Brain tissue oxygen monitoring in traumatic brain injury and major trauma: outcome analysis of a brain tissue oxygen-directed therapy. J Neurosurg 2009;111:672-82.

13 Spiotta AM, Stiefel MF, Gracias VH, et al. Brain tissue oxygendirected management and outcome in patients with severe traumatic brain injury. $J$ Neurosurg 2010;113:571-80.

14 Meixensberger J, Jaeger M, Väth A, et al. Brain tissue oxygen guided treatment supplementing ICP/CPP therapy after traumatic brain injury. J Neurol Neurosurg Psychiatry 2003;74:760-4.

15 Adamides AA, Cooper DJ, Rosenfeldt FL, et al. Focal cerebral oxygenation and neurological outcome with or without brain tissue oxygen-guided therapy in patients with traumatic brain injury. Acta Neurochir 2009;151:1399-409.

16 Martini RP, Deem S, Yanez ND, et al. Management guided by brain tissue oxygen monitoring and outcome following severe traumatic brain injury. J Neurosurg 2009;111:644-9.

17 Green JA, Pellegrini DC, Vanderkolk WE, et al. Goal directed brain tissue oxygen monitoring versus conventional management in traumatic brain injury: an analysis of in hospital recovery. Neurocrit Care 2013;18:20-5. 
18 Okonkwo DO, Shutter LA, Moore C, et al. Brain oxygen optimization in severe traumatic brain injury phase-II: a phase II randomized trial. Crit Care Med 2017;45:1907-14.

19 Chesnut R, Aguilera S, Buki A, et al. A management algorithm for adult patients with both brain oxygen and intracranial pressure monitoring: the Seattle international severe traumatic brain injury consensus conference (SIBICC). Intensive Care Med 2020;46:919-29.

20 Bratton SL, Chestnut RM, Ghajar J, et al. Guidelines for the management of severe traumatic brain injury. $X$. Brain oxygen monitoring and thresholds. J Neurotrauma 2007;24:S65-70.

21 Stocchetti N, Colombo A, Ortolano F, et al. Time course of intracranial hypertension after traumatic brain injury. J Neurotrauma 2007;24:1339-46.

22 Weir J, Steyerberg EW, Butcher I, et al. Does the extended Glasgow outcome scale add value to the conventional Glasgow outcome scale? J Neurotrauma 2012;29:53-8.

23 Jourdan C, Bosserelle V, Azerad S, et al. Predictive factors for 1-year outcome of a cohort of patients with severe traumatic brain injury (TBI): results from the PariS-TBI study. Brain Inj 2013;27:1000-7.

24 Cooper DJ, Rosenfeld JV, Murray L, et al. Decompressive craniectomy in diffuse traumatic brain injury. $N$ Engl J Med 2011;364:1493-502.
25 Steyerberg EW, Wiegers E, Sewalt C, et al. Case-Mix, care pathways, and outcomes in patients with traumatic brain injury in CENTERTBI: a European prospective, multicentre, longitudinal, cohort study. Lancet Neurol 2019;18:923-34

26 Schulz KF, Altman DG, Moher D, et al. Consort 2010 statement: updated guidelines for reporting parallel group randomised trials. Trials 2010;11:32.

27 Badri S, Chen J, Barber J, et al. Mortality and long-term functional outcome associated with intracranial pressure after traumatic brain injury. Intensive Care Med 2012;38:1800-9.

28 Güiza F, Depreitere B, Piper I, et al. Visualizing the pressure and time burden of intracranial hypertension in adult and paediatric traumatic brain injury. Intensive Care Med 2015;41:1067-76.

29 Chesnut RM, Temkin N, Carney N, et al. A trial of intracranial-pressure monitoring in traumatic brain injury. N Engl J Med 2012;367:2471-81.

30 Hutchinson PJ, Kolias AG, Timofeev IS, et al. Trial of decompressive craniectomy for traumatic intracranial hypertension. $N$ Engl J Med 2016;375:1119-30.

31 Leal-Noval SR, Cayuela A, Arellano-Orden V, et al. Invasive and noninvasive assessment of cerebral oxygenation in patients with severe traumatic brain injury. Intensive Care Med 2010;36:1309-17. 